\title{
Mature B cells class switched to IgD are autoreactive in healthy individuals
}

\author{
Kristi Koelsch, ${ }^{1,2}$ Nai-Ying Zheng, ${ }^{1}$ Qingzhao Zhang, ${ }^{1,2}$ Andrew Duty, ${ }^{1,3}$ Christina Helms, ${ }^{1}$ \\ Melissa D. Mathias, ${ }^{1}$ Mathew Jared, ${ }^{1}$ Kenneth Smith, ${ }^{1}$ J. Donald Capra, ${ }^{1,3}$ and Patrick C. Wilson ${ }^{1,2,3}$ \\ ${ }^{1}$ Molecular Immunogenetics Program, Oklahoma Medical Research Foundation, Oklahoma City, Oklahoma, USA. 2Department of Pathology and \\ ${ }^{3}$ Department of Microbiology and Immunology, The University of Oklahoma Health Sciences Center, Oklahoma City, Oklahoma, USA.
}

\begin{abstract}
Determination of the origin and fate of autoreactive B cells is critical to understanding and treating autoimmune diseases. We report that, despite being derived from healthy people, antibodies from $B$ cells that have class switched to IgD via genetic recombination (and thus become class switched to $C \delta[C \delta-C S$ ] cells) are highly reactive to self antigens. Over half of the antibodies from C $\delta$-CS B cells bind autoantigens on human epithelioma cell line 2 (HEp-2) cells or antinuclear antigens, and a quarter bind double-stranded DNA; both groups of antibodies are frequently polyreactive. Intriguingly, some $C \delta-C S$ B cells have accumulated basic residues in the antibody variable regions that mediate anti-DNA reactivity via somatic hypermutation and selection, while other C $\delta$-CS B cells are naturally autoreactive. Though the total percentage was appreciably less than for C $\delta$-CS cells, a surprising $31 \%$ of IgG memory cell antibodies were somewhat autoreactive, and as expected, about $24 \%$ of naive cell antibodies were autoreactive. We interpret these findings to indicate either that autoreactive $B$ cells can be induced to class switch to $\mathrm{IgD}$ or that autoreactive $\mathrm{B}$ cells that use IgD as the $\mathrm{B}$ cell receptor are not effectively deleted. Determination of the mechanism by which the majority of C $\delta$-CS B cells are autoreactive may be important in understanding peripheral tolerance mechanisms and may provide insight into the enigmatic function of the IgD antibody.
\end{abstract}

\section{Introduction}

Autospecific B cells are normally controlled by clonal deletion (1, $2)$, receptor editing $(3,4)$, and clonal anergy $(5,6)$. Despite these mechanisms, B lymphocytes that express surface immunoglobulin that can bind to self antigens are fairly common in the mature human B cell repertoire $(7,8)$. Most knowledge about the mechanisms of $\mathrm{B}$ cell tolerance is derived from experiments with transgenic mice carrying pre-recombined autoantibodies; less is known about the fate of self-reactive B cells in humans. Because autoreactive $B$ cells may be the precursors for the generation of pathological autoantibodies in various autoimmune diseases such as systemic lupus erythematosus (SLE) or rheumatoid arthritis, understanding their fate in healthy humans is critical. Evidence is presented herein that, in healthy people, B cells that are class switched to the $\mathrm{IgD}$ isotype are predominantly autoreactive.

In mature B cells that have not been antigen activated (naive cells), IgM and IgD antibodies are simultaneously encoded by the $\mathrm{C} \mu$ and $\mathrm{C} \delta$ exons via differential mRNA splicing of a single VDJ$\mathrm{C} \mu-\mathrm{C} \delta$ transcript. After activation, naive $\mathrm{B}$ cells can be induced to class switch. Immunoglobulin class switching specializes the function of antibodies through replacement of the $\operatorname{IgM}$ and $\operatorname{IgD}$ gene exons $(C \mu$ and $C \delta)$ with the $\operatorname{IgG}(C \gamma)$, $\operatorname{IgA}(C \alpha)$, or $\operatorname{IgE}(C \varepsilon)$ exons by genetic recombination. However, a small proportion of B cells (1\%-3\%) actually class switch from $\mathrm{C} \mu$ to $\mathrm{C} \delta$ at the genetic level using cryptic switch regions between the $C \mu$ and Co exons and resulting in an $\mathrm{IgM}^{-} \mathrm{IgD}^{+}$phenotype. These are referred to as B cells class switched to C $\delta(C \delta$-CS) cells $(9,10)$ and appear

Nonstandard abbreviations used: ANA, antinuclear antigen; $C \delta-C S$, class switched to $\mathrm{C} \delta$; $\mathrm{CDR}$, complementarity determining region; dsDNA, double-stranded DNA; HEp-2, human epithelioma cell line 2; ssDNA, single-stranded DNA.

Conflict of interest: The authors have declared that no conflict of interest exists. Citation for this article: J. Clin. Invest. 117:1558-1565 (2007). doi:10.1172/JCI27628. to have resulted from an antigen-activated immune response because they can be isolated as GC cells, they are highly clonal (which suggests cell division), and the variable genes have extensive somatic mutations $(11,12)$. C $\delta$-CS B cells also predominantly express $\lambda$-light chains and can differentiate into memory and plasma cells $(13,14)$. The predominant use of $\lambda$-light chains suggests that most secreted IgD antibody in human sera $(\sim 1 \%$ of all antibodies) is from C $\delta$-CS B cells, as serum $\operatorname{IgD}$ is also predominantly $\lambda$-light chain encoded (15). C $\delta$-CS B cells use a subset of immunoglobulin variable region genes long associated with autoreactivity (16) and may have been subjected to receptor editing during $\mathrm{B}$ cell development $(12,16,17)$. These various observations suggest that C $\mathrm{C}$-CS B cells are either generated by a mechanism of immune tolerance or they evade immune tolerance. Herein we set out to determine if indeed C C-CS B cells are autoreactive in healthy humans.

We found that human C $\delta$-CS B lymphocytes were autoreactive, as they encoded antibodies that often bind human epithelioma cell line 2 (HEp-2) cells, antinuclear antigens (ANAs), single-stranded DNA (ssDNA), double-stranded DNA (dsDNA), and they were frequently polyreactive. Consistent with a recent report from Tiller and colleagues, human IgG memory cells had low-intensity autoreactivity and were polyreactive at a surprisingly high frequency (8), although the frequency and intensity of autoantigen binding were significantly less than those of C $\delta$-CS B cells. Interestingly, anti-DNA reactivity was either naturally encoded by the unmutated variable genes or acquired during an immune response via somatic mutations. Thus, anti-DNA antibodies apparently resulting from an immune response might be able to arise in healthy people. These findings provide an important advance in understanding the tolerance mechanisms that control self-reactive B cells that may be disrupted in patients with autoimmune diseases. 
A CD19+tonsil:
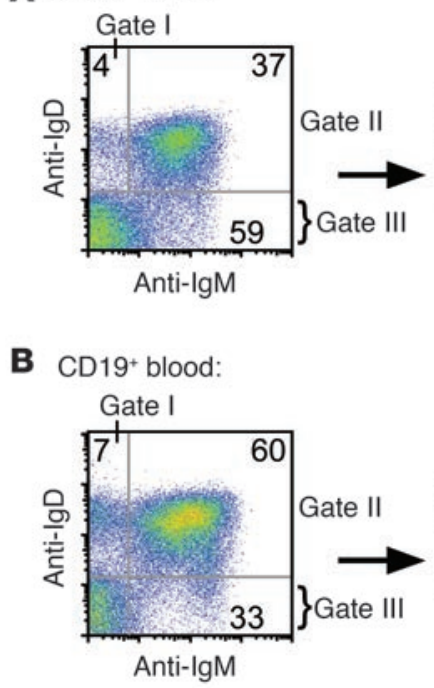

C

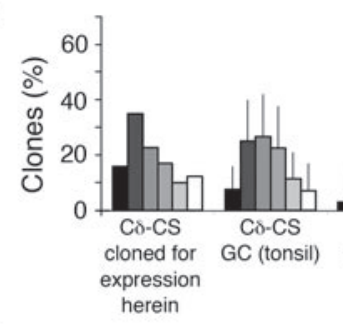

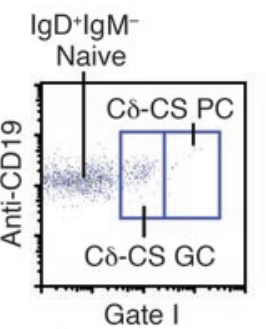

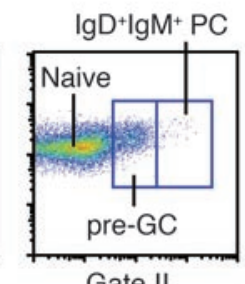

Gate II

Anti-CD38
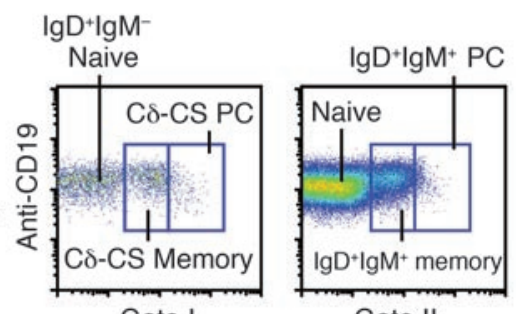

Gate II

Anti-CD27

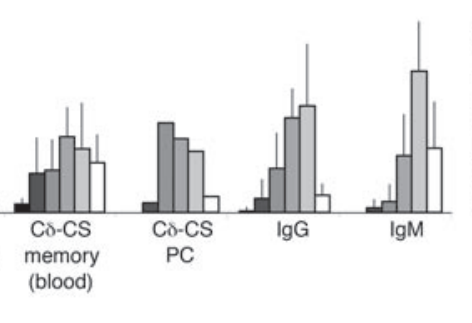

Mutations (no.)

$>40$

31 to 40

$\square 21$ to 30

$\square 11$ to 20

$\square 0$ to 2

D

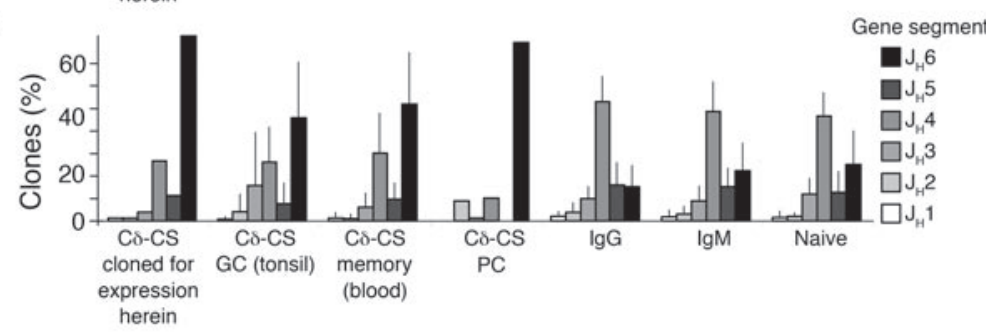

Figure 1

Phenotype of C $\delta$-CS B cells. (A and B) Flow cytometry sorting gates used to isolate the various $B$ cell types analyzed from blood or tonsils, including C $\delta$-CS GC cells $\left(\lg \mathrm{D}+\operatorname{lgM}{ }^{-} \mathrm{CD} 38^{+}\right)$, naive $\mathrm{B}$ cells $\left(\lg \mathrm{D}^{+} \operatorname{lgM} \mathrm{M}^{+}\right.$ and CD38- tonsils or CD27- blood), and memory $\mathrm{B}$ cells $\left(\mathrm{IgD}-\mathrm{CD} 27^{+}\right.$from the "memory" box in B). PC, plasma cell. Analysis of somatic mutation frequency (C) and usage of the $\mathrm{J}_{H} 6$ gene segment (D) demonstrate that all $\mathrm{C} \delta$-CS B cell subsets and those expressed herein are similarly unique from $\lg G$ and $\operatorname{lgM} B$ cells. (C) Shown is the percentage of $V_{H}$ genes with the indicated number of mutations for each subset analyzed. (D) Use of various $J_{H}$ gene segments. Included are transcripts from $625 \mathrm{C} \delta$-CS GC cell variable genes from 11 donors (by donor, $n=44$, $44,57,44,62,34,225,59,16,21$, and 19), $78 \mathrm{C} \delta$-CS plasma cell variable genes from 1 donor, $124 \mathrm{C} \delta$-CS memory cell variable genes from 4 donors (by donor, $n=20,20$, 28 , and 56), $620 \operatorname{lgG~GC}$, memory, and plasma cell variable genes from 14 donors (by donor, $n=18,28,174,40,108,37$, $25,21,18,22,15,24,19$, and 71), 681 IgM GC, memory, and plasma cell variable genes from 18 donors (by donor, $n=18$, $91,51,158,17,10,16,48,30,19,28,11$, $36,29,13,22,20$, and 64 ), and 267 naive variable genes from 6 donors (by donor, $n=47,30,24,15,24$, and 127).

\section{Results}

Determination of the autoantibody specificity of $C \delta-C S B$ cells. C $\delta$-CS $\mathrm{B}$ cells are a unique lineage found in tonsils and blood that are $\mathrm{IgD}^{+} \mathrm{IgM}^{-}$and can be isolated as $\mathrm{GC}\left(\mathrm{CD} 38^{+}\right)$or plasma $\left(\mathrm{CD} 38^{++}\right)$ cells from human tonsils $(11,14)$ (Figure $1 \mathrm{~A}$ ) or as memory cells $\left(\mathrm{CD} 27^{+}\right)$from peripheral blood (13) (Figure 1B). In order to test the specificity of C $\delta$-CS B cells, recombinant monoclonal antibodies were produced from the variable region genes of isolated single cells. We used a modified strategy similar to that described in previous reports $(7,18)$, wherein single B cells were sorted by flow cytometry into 96-well plates and the variable genes amplified by multiplex, single-cell RT-PCR to identify and clone the immunoglobulin heavy-chain and light-chain genes from a random assortment of cells (the variable genes used are listed in Supplemental Table 1; supplemental material available online with this article; doi:10.1172/JCI27628DS1). These variable genes were then cloned into expression vectors and expressed with IgG constant regions in the 293A human cell line. A total of 100 antibodies were produced from $\mathrm{C} \delta$-CS GC B cells ( $\operatorname{IgD}^{+} \operatorname{IgM}^{-} \mathrm{CD} 38^{+}$; Figure 1A) isolated from 3 tonsil donors (by donor, $n=45,34$, and 21 antibodies) and compared with 78 antibodies from naive $\mathrm{B}$ cells from 3 donors $\left(\mathrm{IgD}^{+} \operatorname{IgM}^{+} \mathrm{CD} 38^{-}\right.$; by donor, $n=37,27$, and 14 antibodies) and 64 antibodies from IgG memory cells from 4 donors (IgD ${ }^{-} \mathrm{IgM}^{-} \mathrm{CD} 27^{+}$; by donor, $n=25,12,7$, and 20 antibodies). It is notable that the naive $\mathrm{B}$ cells as defined herein may have contained a minor population of $B$ cells that were transitional cells but that still predominantly represented the naive repertoire of human $B$ cells. It was important to avoid contaminating the naive B cells with $\operatorname{IgM}^{+} \mathrm{D}^{+}$memory cells - because IgM memory cells from humans are infrequently autoreactive (19), contamination would cause us to underestimate the true frequency of autoantibodies detected. In addition to analyzing $\mathrm{CD}^{2} 7^{-}$cells, to further avoid contaminating $\operatorname{IgD}^{+} \operatorname{IgM}^{+}$memory cells, we only used antibodies from the naive cells that had no somatic mutations (i.e., that had less than the background frequency of 1 base exchange per variable gene). The identity of sorted C 8 -CS B cells was verified based on known characteristics of the variable gene repertoire including extensive somatic mutations (Figure 1C) (11) and abundant use of the $\mathrm{J}_{\mathrm{H}} 6$ gene segment (Figure 1D) (16). We were interested to know whether, as predicted by the findings of Klein and colleagues (13), variable genes from C $\delta$-CS peripheral blood memory cells are similar to C8-CS GC and plasma cells. Indeed, randomly cloned 


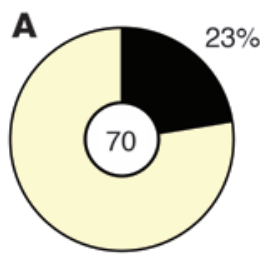

Naive

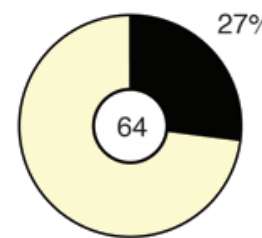

IgG memory

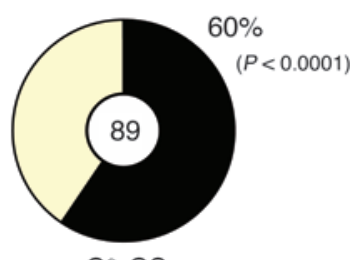

C $\delta$-CS
HEp-2+ and/or ANA ${ }^{+} \quad \square$ Negative

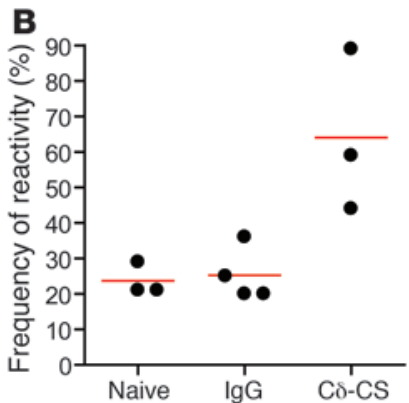

C

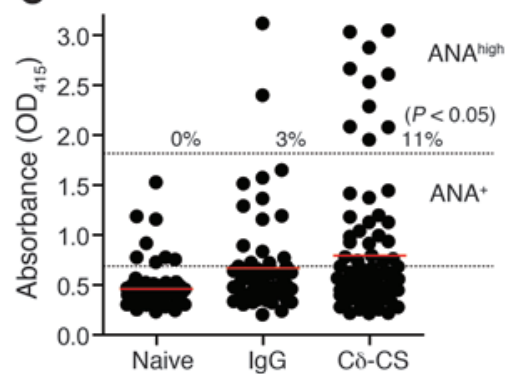

Figure 2

Antibodies from C $\delta$-CS B cells are frequently autoreactive. (A) C $\delta$-CS-derived antibodies are highly reactive to HEp-2 and ANA antigens compared with naive- and IgG-derived antibodies. Antibodies tested included $89 \mathrm{C} \delta$-CS antibodies (from 3 donors, $n=37,18$, and 34 ), 70 naive B cell antibodies (from 3 donors, $n=39,14$, and 17), and 64 antibodies from IgG memory cells (from 4 donors, $n=25,5,12$, and 20). The frequency of HEp-2 reactive antibodies was determined by screening the antibodies by immunofluorescence using commercially prepared HEp-2 slides (Supplemental Figure 1), and the frequency of ANA reactive antibodies using commercial ANA immunosorbent assays (see Methods). Antibodies that bound HEp-2 slides more intensely than negative control serums provided by the manufacturer or that bound ANA more intensely than the mean \pm SD of all naive cell antibodies were considered positive in the 2 assays. Results indicated that the incidence of autoreactivity was significantly higher in $\mathrm{C} \delta$-CS-derived antibodies than in naive- or IgG-derived antibodies $\left(\chi^{2}, P<0.0001\right)$. (B) Frequency of HEp-2 and ANA reactivity by donor. $C \delta$-CS antibodies bound more often than IgG or naive cell-derived antibodies (Student's $t$ test, $P<0.05$ ). (C) Although IgG cells were generally more reactive in the ANA assays than were naive cells, the reactivity was low intensity. In contrast, significantly more C $\delta$-CS antibodies bound to ANA with high intensity. Red lines indicate mean ANA binding absorbances. Dashed lines indicate the thresholds for positive scoring (lower dashed line indicates $A N A^{+}$that is the naive cell mean $\pm S D$; upper dashed line indicates $\left.A N A^{\text {high}}\right)$; the high absorbance level was achieved more commonly for C $\mathrm{C}$-CS antibodies.

variable genes from C $\mathrm{C}$-CS memory cells also had excessive somatic mutations and a preference for use of the autoimmune-associated $\mathrm{J}_{\mathrm{H}} 6$ gene segment (Figure 1, C and D) $(7,16,20)$. As described below, antibodies from C $\delta$-CS GC, naive, and IgG memory cells were tested for reactivity to $\mathrm{HEp}-2$ cells by immunofluorescence and ANA binding by commercial immunosorbent assays and were screened by ELISA for reactivity to DNA or polyreactivity.

Antibodies derived from C $\mathrm{C}-\mathrm{CS} \mathrm{B}$ cells bind self antigens. The HEp-2 Slide assay is the classical clinical diagnostic test used to detect autoreactivity and ANA binding by sera antibodies as seen in autoimmune diseases such as SLE. This assay employs the use of cells from a human epithelioma cell line affixed to a microscope slide, and then detected by immunofluorescence after incubation with the sera (21). To determine if antibodies from CD-CS B cells bound to self antigens at a higher frequency than those from naive or memory B cells, antibodies from each population were tested for reactivity to antigens on the HEp-2 cell line. In addition, all antibodies were screened for binding to ANA using a commercial immunosorbent assay designed for detecting clinical ANA reactivity (QUANTA Lite ANA kit, INOVA Diagnostics Inc.). Antibodies that had detectable binding greater than that of a negative control sera in the HEp-2 immunofluorescence assay were scored as positive. For the ANA assay, an absorbance value was generated that allowed for a more quantitative assessment. Because the naive cells were the control population, we considered antibodies to be ANA positive if they produced absorbencies at the $25-\mu \mathrm{g} / \mathrm{ml}$ concentration that were greater than the mean \pm SD of the absorbance of all naive cell antibodies. The mean \pm SD absorbance of the naive antibodies was $0.4293 \pm 0.2235 \mathrm{OD}_{415}$, so positive tests had absorbencies of 0.6829 or greater. Previous reports have demonstrated that at concentrations of 25 to $50 \mu \mathrm{g} / \mathrm{ml}$, about $70 \%$ of the antibodies from early immature B cells (prior to selection) bind HEp- 2 cell antigens, whereas only $18 \%$ of antibodies from mature naive B cells have appreciable HEp-2 reactivity (7). We found a similar frequency of HEp-2 and ANA reactive antibodies from naive cells tested (16 of 70, or $23 \%$; Figure $2 \mathrm{~A}$ ). Surprisingly, but consistent with a recent report on human IgG antibody reactivity (8), 27\% (17 of 64) of the antibodies from IgG memory B cells were $\mathrm{HEp}-2$ or ANA reactive. In contrast, $60 \%$ (53 of 89 ) of the C8-CS-derived antibodies reacted with HEp-2 or ANA antigens. The increase in frequency of HEp- 2 cell reactive antibodies from C $\mathrm{C}$-CS cells compared with those from naive cells or IgG memory cells was highly significant both when all antibodies were compared as a whole (Figure $2 \mathrm{~A} ; \chi^{2}$, $P<0.0001)$ and when compared by average frequency between donors (Figure 2B; Student's $t$ test, $P<0.05$ versus naive or IgG antibodies).

The CD-CS antibodies also bound to the ANA (Figure 2C) and to HEp-2 antigens (Supplemental Figure 1) with greater intensity than the naive or IgG memory cell antibodies. Eleven percent of the C $\delta$-CS antibodies tested bound ANA with particularly high intensity (ANA ${ }^{\text {high }}$ ) compared with $3 \%$ of the IgG and none of the naive antibodies (Figure $3 \mathrm{C} ; \chi^{2}$, $P=0.05)$. It is notable that approximately half of the IgG antibodies had low-level binding to the ANA antigens that was greater than that of the naive antibodies but not beyond a SD of the mean and so were scored as negative. This slight reactivity of IgG memory cells has recently been show to be due to accumulated somatic mutations (8) and caused an overall increased average absorbance. The $\mathrm{C} \delta$-CS cells in turn had the greatest average ANA binding intensity (Figure $2 \mathrm{C} ; 0.4593 \pm 0.2235 \mathrm{OD}_{415}$ for naive cells, $0.6691 \pm 0.5092 \mathrm{OD}_{415}$ for $\mathrm{IgG}$, and $0.8004 \pm 0.6906 \mathrm{OD}_{415}$ for C $\delta$-CS antibodies). Finally, the HEp-2 autoantigens bound were variable and included cytoplasmic as well as classic ANA patterns (Supplemental Figure 1), thus the self reactivity is not targeted to a single autoantigen. In conclusion, antibodies from C $\delta$-CS B cells commonly bind various antigens on HEp- 2 cells and in commercial ANA assays, demonstrating that they are commonly autoreactive.

Antibodies from buman C $\mathrm{C}$-CS B cells frequently bind to single-stranded and double-stranded DNA. A hallmark of autoreactivity in SLE is the production of antibodies that react to DNA, particularly dsDNA. In order to further evaluate the potential autoreactiv- 

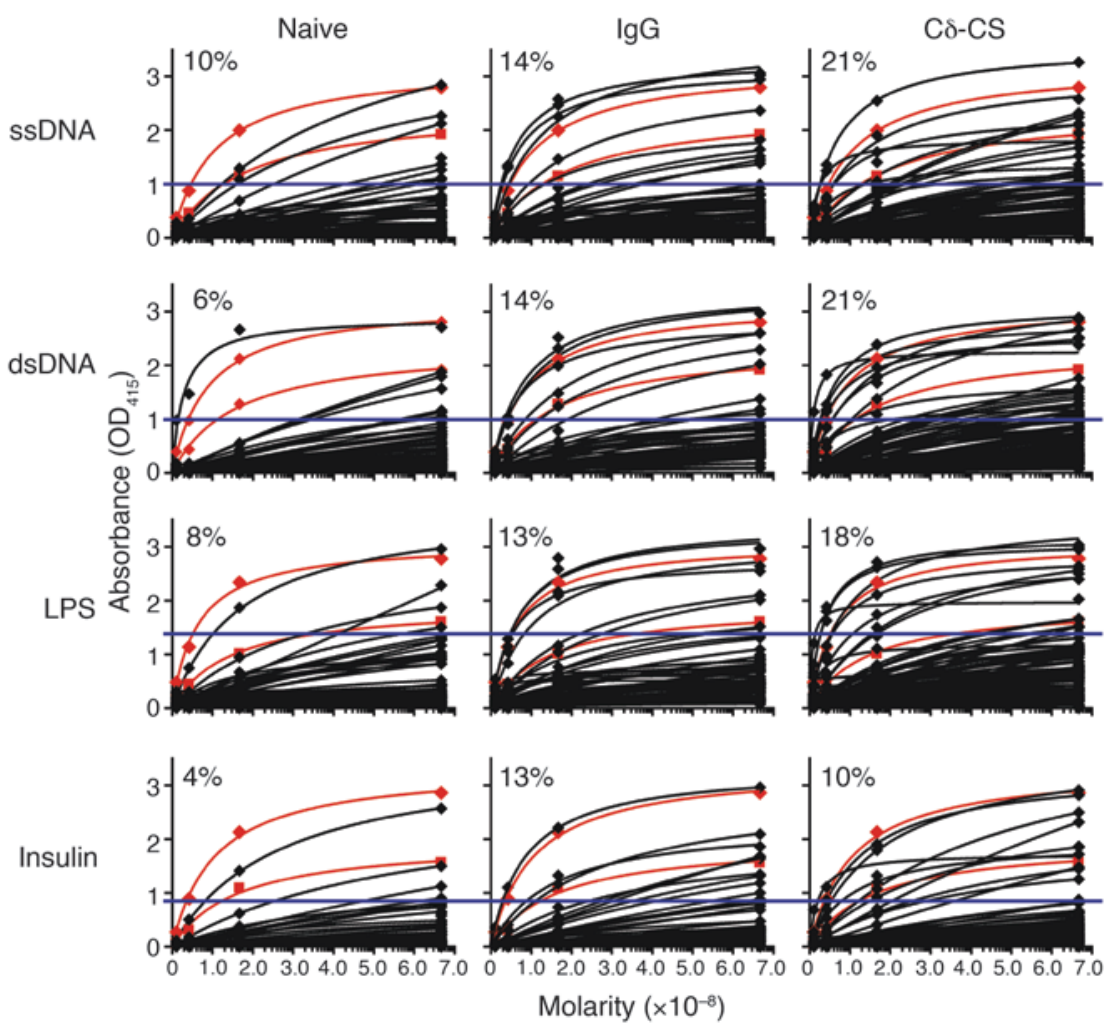

Figure 3

Binding to ssDNA, dsDNA, LPS, or insulin by the expressed antibodies was measured by ELISA. The degree of binding to antigen-coated microtiter plates (absorbance $\left[\mathrm{OD}_{415}\right]$ ) was measured at antibody concentrations of $6.67 \times 10^{-8}, 1.67 \times 10^{-8}, 4.17 \times 10^{-9}$, and $1.04 \times 10^{-9} \mathrm{M}$ (which is $1 \mu \mathrm{g} / \mathrm{ml}$ and three 4 -fold serial dilutions, $x$ axis). All ELISA include $3 \mathrm{H} 9$ (red lines with diamonds) and H241 (red lines with squares) monoclonal antibodies with high and medium anti-DNA or polyreactivity, respectively. The assays were normalized based on the absorbencies of the $3 \mathrm{H} 9$ antibody. Percentages indicate the number of antibodies scored as positive. A total of 78 antibodies from naive B cells isolated from 3 donors (by donor, $n=37,27$, and 14 antibodies) were compared with 100 antibodies from C $\delta$-CS B cells from 3 donors (by donor, $n=45$, 34, and 21 antibodies) and 64 antibodies from IgG memory cells from 4 donors (by donor, $n=25,12,7$, and 20 antibodies). Blue lines represent the approximate positive thresholds determined by calculating the mean \pm 2 SD of the naive antibodies (see Results).

ity of the C $\delta$-CS lineage compared with naive or IgG memory B cells, DNA binding was evaluated by ELISA. DNA binding was tested for the various antibodies at $1 \mu \mathrm{g} / \mathrm{ml}$ and 3 additional 4-fold serial dilutions. Saturation binding curves are presented in Figure 3. Anti-DNA binding affinity was determined by curve fitting. Antibodies with absorbencies calculated for the $1 \mu \mathrm{g} / \mathrm{ml}$ concentration that were 2 SD above the mean absorbance of $95 \%$ of the naive antibodies at that concentration were scored as antiDNA positive (Figure 3). For comparison using the same expression system, the variable genes encoding 2 well characterized antibodies that are commonly used to study anti-DNA reactivity were produced as chimeric mouse/human IgG-K recombinant monoclonals (Figure 3), including $3 \mathrm{H} 9$, used as a transgene to discover receptor editing (3), and H241 (22). Thus, these classic anti-DNA and polyreactive antibodies could be directly compared with identical detection reagents to the human antibodies tested herein. All ELISAs were normalized to the $3 \mathrm{H} 9$ control antibody included on each ELISA plate.
Antibodies reactive to ssDNA are more common in individuals with autoimmune conditions than in healthy individuals; however, they are not diagnostic of a pathological state. As indicated in Figure 3, 8 of 78 (10\%) of the antibodies produced from naive $\mathrm{B}$ cells were reactive to ssDNA. This is consistent with a previous report by Wardemann et al. in which antibodies from 93 naive B cells were analyzed of which several bound ssDNA (7). A greater frequency ( 9 of 64 , or $14 \%$ ) of antibodies from IgG memory cells bound ssDNA. In contrast, $21 \%$ (21 of 100) of the C $\delta$-CS antibodies reacted to ssDNA (Figure $3 ; \chi^{2}, P=0.05$ for C $\delta$-CS versus naive; $P=$ not significant for $C \delta$-CS versus IgG memory).

More important than antibodies to ssDNA are antibodies to dsDNA, as they are more likely indicative of pathology $(23,24)$. Antibodies reactive to dsDNA were less frequent from naive (6\%, or 5 of 78 ) and IgG memory B cells (14\%, 9 of 64 ; Figure 3 ) than from C 8 -CS B cells. Antibodies from C $\delta$-CS cells were 2-fold more likely to bind dsDNA than antibodies from naive cells (21 of 100 , or $21 \%$; $\chi^{2}, P=0.006$ for $\mathrm{C} \delta$-CS versus naive). Again, because IgG antibodies tend to be somewhat polyreactive (8), although $50 \%$ increased, C $\delta$-CS antibodies did not bind dsDNA significantly more frequently. The variability between donors for all assays of autoreactivity is depicted in Figure 4A. As noted previously by Tiller and colleagues, the IgG memory compartment has widely varying levels of anti-DNA reactivity (8). Antibodies that bound dsDNA were also tested for binding to the kinetoplasts of Crithidia luciliae, as this assay is more stringent and is considered the gold standard for detecting dsDNA reactivity in the clinical diagnosis of SLE. In general, there was good correspondence with our results from the ELISA, in that the antibodies with higher affinity for dsDNA by ELISA also bound Crithidia kinetoplasts (data not shown). Thus, similar to the results of the ELISA assays, one of the naive and several of the IgG antiDNA antibodies bound Crithidia kinetoplasts with high intensity and another of the IgG antibodies had only slightly detectable binding. Also as predicted by the ELISA assays, 10 of the C $\delta$-CS antibodies bound Crithidia kinetoplasts and several others had detectable but low-intensity binding. From these experiments, we conclude that $C \delta$-CS B cells are more often reactive to ssDNA and dsDNA than are naive B cells, and C $\mathrm{C}$-CS B cells tend to be more reactive than IgG antibodies.

Antibodies from CS-CS B cells are commonly polyreactive. To determine whether C $\delta$-CS B cells tend to express polyreactive antibodies that interact with multiple self and non-self antigens, we also tested reactivity to recombinant human insulin and lipopolysaccharide from E. coli. As indicated in Figure 4B, significantly more $\mathrm{C} \delta$-CS cell- than naive cell-derived antibodies displayed polyreactivity, but not more than IgG cell-derived antibodies. Whereas 

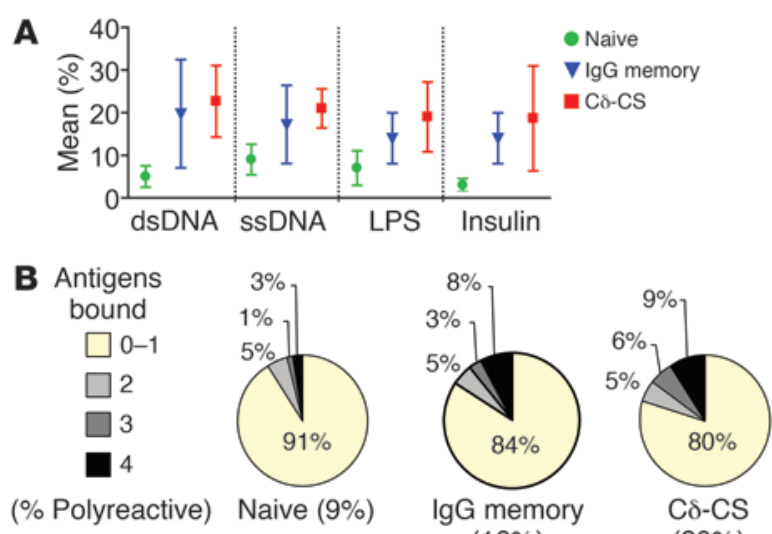

$(16 \%)$

\section{Figure 4}

Variability between donors and frequency of polyreactivity. (A) Mean \pm SEM for donors for the various assays performed. (B) The percentage of antibodies from each group (C $\delta$-CS B cells, naive B cells, or IgG memory $B$ cells) that bound $0-1,2,3$, or all 4 antigens tested (ssDNA, dsDNA, LPS, and insulin). Polyreactivity is defined as the binding of more than 1 antigen. The yellow portion of the chart indicates the percentage that bound up to 1 antigen and so were not polyreactive. Statistical significance $\left(\chi^{2}, P=0.04\right)$ was reached for $C \delta$-CS versus naive $B$ cell antibodies.
$20 \%$ (20 of 100) of the C $\delta$-CS cell-derived antibodies bound at least 2 of the antigens by ELISA, significantly fewer antibodies from naive cells $\left(9 \%\right.$, or 7 of $\left.79 ; \chi^{2}, P=0.039\right)$ interacted with 2 or more antigens. For IgG memory cells the incidence of polyreactivity was less than that of C 8 -CS (16\% versus $20 \%$, or 10 of 64 versus 20 of 100); however, significance was not reached. In conclusion, antibodies from B cells that had class switched to IgD or IgG were commonly polyreactive.

Altogether, considering the high frequency of HEp-2 and ANA reactivity and the frequency of anti-DNA and polyreactivity, we concluded that $56 \%$ C $\delta$-CS antibodies were autoreactive. Thus C $\delta$-CS cells were about 2 -fold more frequently autoreactive than naive cell antibodies ( $24 \%$ autoreactive, $\left.\chi^{2}, P<0.0001\right)$ and 1.8 -fold more frequent than IgG cell-derived antibodies (31\% autoreactive, $\left.\chi^{2}, P<0.001\right)$. From this analysis, we concluded that C $\delta$-CS B cells are usually autoreactive.

IgD class switching occurs both in B cells with natural autoantibodies and for cells with autoreactivity generated by somatic hypermutations. It is possible that a class switch to $\operatorname{IgD}$ occurred only for autoreactivity introduced during immune responses due to the excessive somatic mutations characteristic of CD-CS B cells (Figure 1C) (11). A recent report from Tiller and colleagues found a surprisingly high frequency of IgG memory B cells that are polyreactive, and the reactivity was typically caused by accumulated somatic hypermutations (8). Alternatively, it is plausible that a class switch to IgD may have occurred prior to somatic mutation and independent of a normal GC reaction. Consistent with this conjecture, we have previously demonstrated that C $\delta$-CS B cells accumulate targeted somatic mutations that disrupt the natural autoreactivity of antibodies encoded by the $\mathrm{V}_{\mathrm{H}} 4-34$ gene segment (16). This finding suggested that the accumulation of excessive somatic hypermutations in the variable genes of C $\delta$-CS B cells may have resulted from selective pressure to alter amino acids causing natural autoreactivity as well as increasing affinity to foreign antigens. In order to gain insight into the origin of autoreactive $\mathrm{B}$ cells that are class switched to $\mathrm{IgD}$, we characterized the role of somatic mutation in mediating the autoreactivity. The fact that 11 of 100 (11\%) of the C $\delta$-CS antibodies expressed were from unmutated variable genes allowed us to determine if $\mathrm{B}$ cells that were class switched to IgD could have natural autoreactivity (not due to mutations). Note that the variable genes encoding these transcripts were otherwise similar to that of most C $\delta$-CS antibodies with preferential use of the $\mathrm{J}_{\mathrm{H}} 6$ gene segment, long complementarity determining regions 3 [CDR3s], and $\lambda$-light chain usage (data not shown). As indicated in Figure 5A, the frequencies of HEp-2 antigen autoreactivity, binding to dsDNA, and polyreactivity were similar in mutated and unmutated antibodies from the C $\delta$-CS B cells and were higher than the frequency of autoantibodies from naive cells and IgG memory cells. This analysis also provided verification that these $C \delta$-CS clones with unmutated variable-region genes were not contaminating naive cells. In addition, comparison of anti-DNA titers with the frequency of amino acid replacements indicated that there was no correlation between the affinity for dsDNA and the accumulation of somatic mutations, as titers even tended to be higher for the several clones without mutations (Figure 5B). From these observations, we concluded that cells class switched to IgD can express naturally (germline-encoded) autoreactive antibodies independent of somatic hypermutation.

Arginine is considered the most important residue for antiDNA binding $(25,26)$. The antibodies with natural reactivity to DNA and number of the other antibodies had arginines introduced to the CDR3 by VDJ recombination (Cס-CS clones 1 and 2 were germline, also note that arginines were introduced in the CDRs of clones 4, 6, 8, 10, 11, 12, 14, and 21; Supplemental Figure 2 ). An important observation was that most of the C $\delta$-CS clones with anti-DNA reactivity also had arginines that were introduced because of codon alterations that resulted from somatic mutation (Supplemental Figure 2). In fact, 15 of 22 (68\%) of these C $\delta$-CS dsDNA binding clones had as many as 7 arginines introduced by mutation into the heavy and/or light chains (clone 18 had the most arginines introduced; Supplemental Figure 2). This suggested that the autoreactivity we observed may have arisen as a result of somatic mutations introduced during immune responses. In order to explore this possibility, we generated and expressed the unmutated counterparts of the variable genes of 2 of the anti-DNA antibodies from C 8 -CS cells (clones 14 and 17; Figure $5 \mathrm{~B}$ ). Neither of the unmutated variants bound dsDNA, demonstrating that the anti-DNA reactivity that we observed arose as a result of somatic hypermutation (Figure 5C). Thus, anti-DNA antibodies that eventually cause autoimmune disease may commonly arise as the result of antigen-specific immune responses in healthy people. Taken together, these analyses suggest that either C $\delta$-CS B cells can be either naturally autoreactive or that their autoreactivity can arise due to amino acid changes introduced by somatic hypermutation. 

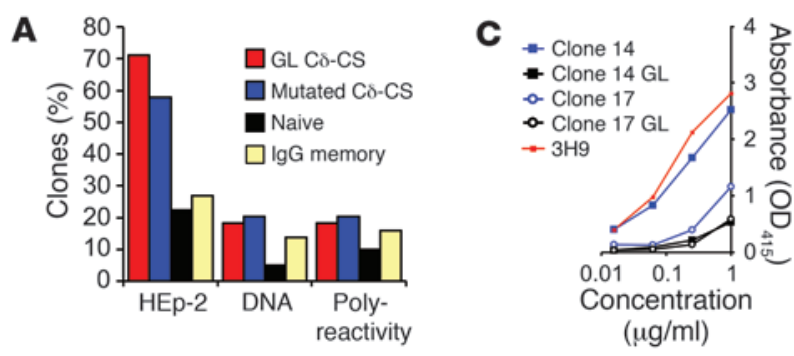

B

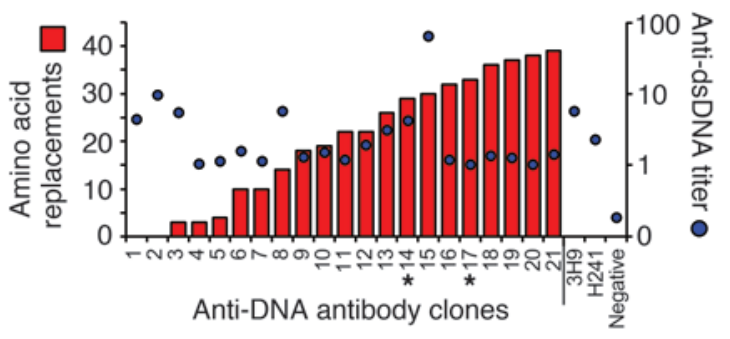

Figure 5

C $\delta$-CS can occur for cells that express germline (natural) autoreactive antibodies as well as those that have acquired autoreactivity via somatic mutations. (A) CD-CS antibodies encoded by unmutated (germline) variable genes display levels of HEp-2 autoreactivity, DNA binding, and polyreactivity similar to those from somatically mutated variable genes. Shown are the percentages of antibodies from each subpopulation. (B) Binding curves of $1 \mu \mathrm{g} / \mathrm{ml}$ anti-DNA antibodies from CD-CS B cells to DNA were used to calculate absorbencies (blue dots). Comparison of these absorbencies with the frequency of amino acid replacements (red bars) showed that there is no correlation between the affinity for dsDNA and the accumulation of somatic mutations. The variable genes of clones 14 and 17 (asterisks) were reverted to their germline sequences to determine whether the somatic mutations might cause DNA binding. (C) DNA binding is lost when 2 anti-DNA C $\delta$-CS antibodies (clone 14, blue square; clone 17, blue circle) were expressed from variable genes reverted to the germline unmutated sequences (clone 14, black square; clone 17, black circle). Anti-DNA binding was evaluated by ELISA (absorbance [OD 415$]$ ) relative to the control $3 \mathrm{H} 9$ monoclonal antibody with high affinity for DNA (red line). GL, germline.

\section{Discussion}

Several possibilities exist for the high rates of autoreactivity found in C $\delta$-CS B cells. It is believed that class switching to $\operatorname{IgD}$ and the resulting $\mathrm{C} \delta$-CS $\mathrm{B}$ cells arise during $\mathrm{GC}$ reactions because they typically express highly mutated variable genes, they are clonally expanded, and they can only be isolated as GC, memory, or plasma cells $(11,13,14,16)$. We were not able to discern whether $B$ cells that are autoreactive are induced to class switch to $\operatorname{IgD}$ or whether IgDexpressing B cells that are autoreactive are preferentially selected. In either case, a number of hypothetical models can be envisioned. First, there may be a directed mechanism by which the cryptic $C \delta$ switch region is targeted in activated human $B$ cells that are self reactive. Such a mechanism may have evolved to rescue autoreactive B cells for some specialized or reduced role in immunity involving IgD. It is interesting to consider that the elusive function of $\operatorname{IgD}$ may be uniquely involved in self tolerance (reviewed in ref. 15). However, analyses of transgenic mice expressing IgD or IgM alone as autoantibodies found little difference between $\operatorname{IgD}$ and IgM in B cell tolerance or negative selection (27). In fact, a number of studies suggest that $\operatorname{IgD}$ augments $\operatorname{IgM}$ signaling to positively select B cells into the functional repertoire (reviewed in refs. 15 and 28). With these studies in mind, several other models can be envisioned in which IgD does not have a specific role in tolerance. One possibility is that autoreactive B cells may be inhibited from class switching to the downstream Ig isotypes (i.e., IgG, IgA, IgE), leaving only the cryptic $\mathrm{C} \delta$ switch region available for switch in cells that previously bound self antigen. This inhibition of class switch may occur as a manifestation of anergy. Both of the above models are interesting because they each suggest that tolerizing mechanisms can directly control immunoglobulin class switch.

An alternative hypothesis is that class switch to $\operatorname{IgD}$ is random (i.e., any cell can switch), and that selection of CD-CS B cells occurs after class switch, causing preferential survival of the autoreactive clones. For example, it has been suggested that, compared with IgM receptors alone, expression of IgD provides preferential survival (29) and recruitment of B cells to immune responses that might allow for rescue by chronic stimulation with self antigen (30). Another possible explanation is that, when stimulated by self antigens or without the accessory signals that are normally required, expression of $\mathrm{IgD}$ only on the $\mathrm{B}$ cell surface may not result in $\mathrm{B}$ cell death. Elucidation of the actual mechanism that causes C $\delta$-CS B cells to be autoreactive might provide important insights into the role of $\mathrm{IgD}$ as a B cell receptor and into peripheral mechanisms of immune tolerance.

IgG memory B cells commonly express low-affinity autoantibodies (8), mostly as a result of changes from accumulated somatic mutations. In our study, $31 \%$ of IgG antibodies had detectable autoreactivity. It is notable that recent analyses have found that antibodies from $\mathrm{IgM}^{+}$memory B cells were rarely autoreactive, but when autoreactivity was found it was caused by accumulated somatic mutations (19). Similarly, most autoreactivity seen in $\mathrm{IgG}^{+}$memory B cells from healthy people is caused by somatic mutations (8). It is known that $\mathrm{IgG}^{+}$memory B cells have accumulated approximately 2 -fold the frequency of point mutations as IgM memory cells (31) and correspondingly are more often autoreactive (8). C $\delta$-CS B cells in turn have accumulated the most point mutations, averaging $21 \pm 7$ base exchanges per $V_{H}$ gene compared with $14 \pm 5$ per gene for IgG and $8 \pm 4$ mutations per gene for IgM GC and memory cells (Figure 1C). Concurrently, C $\delta$-CS B cells are also the most often autoreactive, though unlike IgG and IgM memory cells, the $10 \%$ of C 8 -CS B cells that are not mutated are still autoreactive (Figure 5A). The mildly mutated IgM memory compartment may be more reminiscent of the naive $B$ cell repertoire that is then further selected after antigen interaction, thus reducing the overall frequency of autoantibodies (19). In contrast, excessively activated B cells that accumulate more mutations and eventually class switch may be selected to avoid pathological autoreactivity, as evidenced by preferential differentiation to C $\mathrm{C}$-CS B cells, but nevertheless, further divergence from germline does result in an increased frequency of IgG memory B cells that are autoreactive and polyreactive. Culton and colleagues recently reported that there is a selective checkpoint that removes autoreactive $\mathrm{B}$ cell clones during the transition to plasma cells (32). This checkpoint could suppress the ability of autoreactive IgG memory cells to become antibody secreting cells in healthy persons. One final conjecture is that there may be qualitative differences between autoreactive IgG and C $\delta$-CS antibodies, as suggested by the greater intensity of binding of $\mathrm{C} \delta$ - $\mathrm{CS}$ antibodies to ANA (Figure 2C) and HEp-2 antigens (Supplemental Figure 1). For example, the low-intensity potential autoreactivity of the IgG 
polyreactive antibodies might be absorbed or masked by nonspecific interaction with other molecules in serum and therefore not represent a significant threat to self but still be a threat to the antigen for which they were selected to bind with high affinity. In contrast, specificities that are selected in C $\delta$-CS B cells may be more blatantly autoreactive. A more in-depth analysis of IgG specificity compared with C $\mathrm{C}$-CS specificity and affinity to autoantigens is required to clarify this possibility.

In conclusion, the findings herein demonstrate that C 8 -CS (IgDonly) B cells have a high rate of autoreactivity in healthy persons. Characterization of the mechanism that causes the majority of C $\delta$-CS B cells to be autoreactive may provide important advances in our understanding of peripheral mechanisms of immune tolerance and perhaps a better understanding of the elusive function of the $\operatorname{IgD}$ isotype.

\section{Methods}

B lymphocyte isolation and cytometry. B lymphocytes were isolated from human tonsils or blood as previously described $(11,14,31)$. All tissues and blood were isolated following established NIH guidelines with institutional review board approval from the Oklahoma Medical Research Foundation Institutional Review Board (IRB) (blood and tonsils) and The University of Oklahoma Health Sciences Center IRB (tonsils). Tonsils were obtained from The University of Oklahoma Children's Hospital during routine tonsillectomy and blood was from the Oklahoma Blood Institute, Oklahoma City, Oklahoma, USA. Use of tonsil tissue and blood was exempt from written consent. All tonsil donors were healthy children with a history of inflamed tonsils (but without any current inflammation). Blood was from young adult volunteers. Briefly, individual cells were teased from the tonsils. B cell-enriched fractions were purified using RosetteSep reagents (StemCell Technologies Inc.) and a lymphoprep gradient (CellGro; Mediatech Inc.) with sheep rbc added. $B$ cells were then enriched to near purity by magnetic bead depletion using the Miltenyi Biotec B Cell Isolation kit II. Naive B cells were isolated as $\mathrm{IgD}^{+} \mathrm{IgM}^{+} \mathrm{CD}^{-} 8^{-}$(tonsil) or $\mathrm{CD}^{-} 7^{-}$(blood) and C $\mathrm{C}-\mathrm{CS} \mathrm{B}$ cells were isolated as $\operatorname{IgD}^{+} \operatorname{IgM}^{-} \mathrm{CD}_{38}{ }^{+}$, and peripheral blood IgG memory cell transcripts were amplified by RT-PCR from sorted total memory cells $\left(\mathrm{IgD}^{-} \mathrm{IgM}^{-} \mathrm{CD} 27^{+}\right.$; Figure $\left.1 \mathrm{~B}\right)$ using a Dako Cytomation MoFlo or a BD FACSAria flow cytometer. Plates of both $\operatorname{IgK}^{+}$and $\operatorname{Ig} \lambda^{+}$from naive and C $\delta$-CS cells were sorted from 2 of the donors. Because the IgK and Ig $\lambda$ fractions were similar for all assays, the fractions were grouped for the analyses herein. As predicted, most C $\delta$-CS B cells were Igא negative $\left(\operatorname{Ig} \lambda^{+}\right)$. Bulk cells of the 3 phenotypes were sorted and resorted into 96-well PCR plates to ensure purity of single cells (98\%-99\% purity detected on sort of the single cells). Antibodies used for flow cytometry were anti-IgM-conjugated to APCs (Southern Biotechnology Associates), anti-IgD-conjugated to PE (BD), biotinylated anti-CD38 followed by streptavidin-conjugated to tricolor (Invitrogen), anti-IgK-conjugated to FITC (Invitrogen), and anti-CD27-conjugated to PE (Invitrogen).

Single-cell RT-PCR and PCR. Single B cells were sorted into PCR plates containing $10 \mathrm{mM}$ Tris-HCL with 40 units/ $\mu \mathrm{l}$ of RNase inhibitor (Promega). The plates were immediately frozen on dry ice and stored at $-80^{\circ} \mathrm{C}$. Each cell was amplified in a 1-step RT-PCR reaction (QIAGEN) using a cocktail of sense primers specific to the leader regions and antisense primers specific to the $\mathrm{C} \mu$ - (naive), $\mathrm{C} \delta$ - (Cס-CS), or $\mathrm{C} \gamma$-constant regions for heavy chains and $\mathrm{C} \kappa$-constant or $\mathrm{C} \lambda$-constant regions for the light chains. One microliter from each RT-PCR reaction was amplified in separate PCR reactions for the individual heavy- and light-chain gene families using nested primers. All primer sequences have been previously published $(7,18)$ except for the anti-C $\delta$ primers (external, 5'-GTGTCTGCACCCTGATATGATGG-3', nested, $5^{\prime}$-GGGAACACATCCGGAGCCTTG-3') and the anti-C $\gamma$ primers (external, 5'-TCTTGTCCACCTTGGTGTTGCT-3' , nested, 5'-AGGTGCTCTTGGAGGAGGGT-3'). PCR products were sequenced (Applied Biosystems 3730 DNA Analyzer). Upon identification of the variable genes, sense primers unique to the particular variable genes and antisense primers binding the particular junctional genes were used to amplify $1 \mu$ l of the RT-PCR reaction to incorporate restriction sites at the ends of the variable genes for cloning and expression. Some of the variable gene sequences presented for comparison in Figure 1, C and D, were from historical data previously published by our laboratory $(12,16,33,34)$. To generate the reverted variable genes for the experiments in Figure 5, we used strandoverlap extension PCR of unmutated variable-heavy, variable-light, and $\mathrm{J}$ templates and overlapping primers encoding the CDR3 regions with gene-specific $V$ and $J$ primers.

Recombinant monoclonal antibody expression. Following purification and digestion, the amplified cDNAs of the antibody variable genes from each single cell were cloned into expression vectors containing human $\operatorname{IgG}$, Igא, or Ig $\lambda$ constant regions as previously described (7). Maxi prep plasmids Plasmid Maxi Kit (QIAGEN) containing the heavy- and lightchain Ig genes were cotransfected into the 293A cell line using calcium phosphate or Roche Applied Science FuGENE 6 transfection reagent as per the manufacturer's suggested protocol. Transfected 293A cells were allowed to secrete antibodies in serum-free DMEM supplemented with 1\% Nutridoma-SP (Roche) for 4 to 5 days. Antibodies were purified using immobilized protein A columns (Pierce). Proper antibody expression and purity were verified by polyacrylamide gel electrophoresis, and purified antibody concentrations were determined using the EZQ Protein Quantitation Kit (Invitrogen).

ELISA and HEp-2 analyses for autoreactivity. To screen the expressed antibodies for DNA reactivity or polyreactivity, ELISA microtiter plates (Costar; Corning Inc.) were coated with $10 \mu \mathrm{g} / \mathrm{ml}$ calf thymus ssDNA or dsDNA (Invitrogen), LPS (Sigma-Aldrich), or recombinant human insulin (Fitzgerald). $\gamma$-chain-specific goat anti-human IgG-peroxidase conjugate (Jackson ImmunoResearch Laboratories Inc.) was used to detect binding of the recombinant antibodies, followed by development with horseradish peroxidase substrate (Bio-Rad). Absorbances were measured at $\mathrm{OD}_{415}$ on a microplate reader (Molecular Devices).

The antibodies were screened for HEp-2 reactivity by immunofluorescence using commercial HEp-2 slides as per the manufacturers suggested protocol (BION Enterprises Ltd.). HEp-2 slides were analyzed using a Zeiss Axioplan II fluorescent microscope. The antibodies were screened for ANA binding using the QUANTA Lite ANA kit (INOVA Diagnostics Inc.) and detected using goat anti-human $\operatorname{IgG}(\gamma$-chain specific) peroxidase-conjugate (Jackson ImmunoResearch Laboratories Inc.) followed by development with horseradish peroxidase substrate (Bio-Rad). All HEp-2 and ANA reactivity was compared to positive control sera from a lupus patient and to nonreactive sera from a healthy blood donor. The QUANTA Lite ANA assays were normalized to an in house $\mathrm{ANA}^{+}$monoclonal antibody (mAb: 050504P181L+B09).

Statistics. Statistical analyses were performed using Microsoft Excel 2003 or GraphPad Prism 4 software. For the analyses in Figure 1, A and B, and Figure 2B, 2-tailed Student's $t$ tests were performed. For the analyses in Figure 2, A and C, Figure 3, and Figure 4B, $\chi^{2}$ tests were performed.

\section{Acknowledgments}

We thank Judith A. James for critically reading the manuscript and providing valuable suggestions. We thank Larry Wysocki for providing, and Martin Weigert and David Stollar for allowing us to use, the $3 \mathrm{H} 9$ and $\mathrm{H} 241$ anti-DNA positive control monoclones. Technical assistance was provided by Leni Abraham and 
Sheryl Christopherson from the Oklahoma Medical Research Foundation (OMRF) DNA Sequencing Facility, the staff of the OMRF Imaging Core Facility, and Jacob Bass and Diana Hamilton in the OMRF Flow Cytometry Core Facility. We also thank the University of Oklahoma Children's Hospital and particularly Sarah Johnson and Jesus Medina for making tonsil specimens available. Lisa Ridgeway provided clerical assistance. This work was funded in part by NIH grants P20RR018758-01 and P20RR15577-02 to P.C. Wilson.

1. Nemazee, D.A., and Burki, K. 1989. Clonal deletion of B lymphocytes in a transgenic mouse bearing antiMHC class I antibody genes. Nature. 337:562-566.

2. Hartley, S.B., et al. 1991. Elimination from peripheral lymphoid tissues of self-reactive B lymphocytes recognizing membrane-bound antigens. Nature. 353:765-769.

3. Gay, D., Saunders, T., Camper, S., and Weigert, M. 1993. Receptor editing: an approach by autoreactive B cells to escape tolerance. J. Exp. Med. 177:999-1008.

4. Tiegs, S.L., Russell, D.M., and Nemazee, D. 1993. Receptor editing in self-reactive bone marrow B cells. J. Exp. Med. 177:1009-1020.

5. Nossal, G.J. 1983. Cellular mechanisms of immunologic tolerance. Annu. Rev. Immunol. 1:33-62.

6. Goodnow, C.C., et al. 1988. Altered immunoglobulin expression and functional silencing of selfreactive B lymphocytes in transgenic mice. Nature. 334:676-682.

7. Wardemann, H., et al. 2003. Predominant autoantibody production by early human B cell precursors. Science. 301:1374-1377.

8. Tiller, T., et al. 2007. Autoreactivity in human $\mathrm{IgG}(+)$ memory B cells. Immunity. 26:205-213.

9. White, M.B., Word, C.J., Humphries, C.G., Blattner, F.R., and Tucker, P.W. 1990. Immunoglobulin D switching can occur through homologous recombination in human B cells. Mol. Cell. Biol. 10:3690-3699.

10. Yasui, H., Akahori, Y., Hirano, M., Yamada, K., and Kurosawa, Y. 1989. Class switch from mu to delta is mediated by homologous recombination between sigma mu and sigma mu sequences in human immunoglobulin gene loci. Eur. J. Immunol. 19:1399-1403.

11. Liu, Y.J., et al. 1996. Normal human $\operatorname{IgD}^{+} \operatorname{IgM}^{-}$germinal center B cells can express up to 80 mutations in the variable region of their IgD transcripts. Immunity. 4:603-613.

12. Wilson, P.C., et al. 2000. Receptor revision of immunoglobulin heavy chain variable region
Received for publication December 12, 2005, and accepted in revised form March 27, 2007.

Address correspondence to: Patrick C. Wilson, Molecular Immunogenetics, Oklahoma Medical Research Foundation, 825 NE 13th St., Oklahoma City, Oklahoma 73104, USA. Phone: (405) 271-7393, ext.34556; Fax: (405) 271-8237; E-mail: wilsonp@omrf.ouhsc.edu.

Kristi Koelsch and Nai-Ying Zheng contributed equally to this work. genes in normal human B lymphocytes. J. Exp. Med. 191:1881-1894.

13. Klein, U., Rajewsky, K., and Kuppers, R. 1998. Human immunoglobulin ( $\mathrm{Ig}) \mathrm{M}+\mathrm{IgD}+$ peripheral blood B cells expressing the CD27 cell surface antigen carry somatically mutated variable region genes: CD27 as a general marker for somatically mutated (memory) B cells. J. Exp. Med. 188:1679-1689.

14. Arpin, C., et al. 1998. The normal counterpart of IgD myeloma cells in germinal center displays extensively mutated $\mathrm{IgVH}$ gene, Cmu-Cdelta switch, and lambda light chain expression. J. Exp. Med. 187:1169-1178.

15. Preud'homme, J.L., et al. 2000. Structural and functional properties of membrane and secreted $\operatorname{IgD}$. Mol. Immunol. 37:871-887.

16. Zheng, N.Y., et al. 2004. Human immunoglobulin selection associated with class switch and possible tolerogenic origins for C $\delta$ class-switched B cells. J. Clin. Invest. 113:1188-1201. doi:10.1172/ JCI200420255.

17. van der Burg, M., et al. 2002. Biased Iglambda expression in hypermutated IgD multiple myelomas does not result from receptor revision. Lenkemia. 16:1358-1361.

18. Meffre, E., et al. 2004. Surrogate light chain expressing human peripheral B cells produce self-reactive antibodies. J. Exp. Med. 199:145-150.

19. Tsuiji, M., et al. 2006. A checkpoint for autoreactivity in human IgM+ memory B cell development. J. Exp. Med. 203:393-400.

20. Meffre, E., et al. 2000. Circulating human B cells that express surrogate light chains and edited receptors. Nat. Immunol. 1:207-213.

21. Bradwell, A.R., Stokes, R.P., and Johnson, G.D. 1995. Atlas of HEp-2 patterns. The Binding Site Ltd. Birmingham, United Kingdom. 9-37.

22. Stollar, B.D., Zon, G., and Pastor, R.W. 1986. A recognition site on synthetic helical oligonucleotides for monoclonal anti-native DNA autoantibody. Proc. Natl. Acad. Sci. U. S. A. 83:4469-4473.

23. Koffler, D., Carr, R.I., Agnello, V., Fiezi, T., and
Kunkel, H.G. 1969. Antibodies to polynucleotides: distribution in human serums. Science. 166:1648-1649.

24. Radic, M.Z., and Weigert, M. 2004. Intricacies of anti-DNA autoantibodies. J. Immunol. 172:3367; author reply 3367-3368.

25. Shlomchik, M., et al. 1990. Anti-DNA antibodies from autoimmune mice arise by clonal expansion and somatic mutation. J. Exp. Med. 171:265-292.

26. Wellmann, U., et al. 2005. The evolution of human anti-double-stranded DNA autoantibodies. Proc. Natl. Acad. Sci. U. S. A. 102:9258-9263.

27. Brink, R., et al. 1992. Immunoglobulin M and D antigen receptors are both capable of mediating B lymphocyte activation, deletion, or anergy after interaction with specific antigen. J. Exp. Med. 176:991-1005.

28. Geisberger, R., Lamers, M., and Achatz, G. 2006. The riddle of the dual expression of IgM and IgD. Immunology. 118:429-437.

29. Brink, R., Fulcher, D.A., Goodnow, C.C., and Basten, A. 1994. Differential regulation of early and late stages of B lymphocyte development by the $\mathrm{mu}$ and delta membrane heavy chains of Ig. Int. Immunol. 6:1905-1916.

30. Roes, J., and Rajewsky, K. 1993. Immunoglobulin $\mathrm{D}(\mathrm{IgD})$-deficient mice reveal an auxiliary receptor function for $\operatorname{IgD}$ in antigen-mediated recruitment of B cells. J. Exp. Med. 177:45-55.

31. Pascual, V., et al. 1994. Analysis of somatic mutation in five B cell subsets of human tonsil. J. Exp. Med. 180:329-339.

32. Culton, D.A., et al. 2006. Early preplasma cells define a tolerance checkpoint for autoreactive B cells. J. Immunol. 176:790-802.

33. Wilson, P.C., et al. 1998. Somatic hypermutation introduces insertions and deletions into immunoglobulin V genes. J. Exp. Med. 187:59-70.

34. Zheng, N.Y., Wilson, K., Jared, M., and Wilson, P.C. 2005. Intricate targeting of immunoglobulin somatic hypermutation maximizes the efficiency of affinity maturation. J. Exp. Med. 201:1467-1478. 